\title{
Role of attractive forces in tapping tip force microscopy
}

\author{
Kyhle, Anders; Sørensen, Alexis Hammer; Bohr, Jakob
}

Published in:

Journal of Applied Physics

Link to article, DOI:

10.1063/1.365194

Publication date:

1997

Document Version

Publisher's PDF, also known as Version of record

Link back to DTU Orbit

Citation (APA):

Kyhle, A., Sørensen, A. H., \& Bohr, J. (1997). Role of attractive forces in tapping tip force microscopy. Journal of Applied Physics, 81(10), 6562-6569. https://doi.org/10.1063/1.365194

\section{General rights}

Copyright and moral rights for the publications made accessible in the public portal are retained by the authors and/or other copyright owners and it is a condition of accessing publications that users recognise and abide by the legal requirements associated with these rights.

- Users may download and print one copy of any publication from the public portal for the purpose of private study or research.

- You may not further distribute the material or use it for any profit-making activity or commercial gain

- You may freely distribute the URL identifying the publication in the public portal

If you believe that this document breaches copyright please contact us providing details, and we will remove access to the work immediately and investigate your claim 


\title{
Role of attractive forces in tapping tip force microscopy
}

\author{
Anders Kühle, ${ }^{\text {a) }}$ Alexis H. Sørensen, and Jakob Bohr \\ Department of Physics, Building 307, Technical University of Denmark, DK-2800 Lyngby, Denmark
}

(Received 14 November 1996; accepted for publication 3 February 1997)

\begin{abstract}
We present experimental and numerical results demonstrating the drastic influence of attractive forces on the behaviour of the atomic force microscope when operated in the resonant tapping tip mode in an ambient environment. It is often assumed that tapping is related to repulsive interaction. In contrast, we find that in general the attractive forces are the most dominant interaction in this mode of operation. We show that attractive forces in combination with the repulsive elastic type of forces cause points of instability in the parameter space constituted by: the cantilever swing amplitude, the frequency bias point, and the distance between the fixed end of the cantilever and the sample. These points of instability can result in disturbances during image acquisition on hard elastic surfaces. (C) 1997 American Institute of Physics. [S0021-8979(97)00310-1]
\end{abstract}

\section{INTRODUCTION}

The number of applications of the scanning probe microscope is steadily increasing along with the development of new modes of operation. The atomic force microscope $(\mathrm{AFM})^{1-4}$ with a resonant vibrating cantilever ${ }^{5,6}$ has become widely used. In the vibrating cantilever mode, the cantilever is excited close to its resonance frequency $f_{c}$ by an external signal that is applied to a piezoelectric ceramic attached to the base of the cantilever. Two modes can be distinguished by the magnitude of the cantilever swing amplitude $a$.

In the low amplitude mode $(a<5 \mathrm{~nm})$, often referred to as the (true) non-contact mode, the swing amplitude is so small that the end of the tip is embedded in the attractive force fields, e.g., van der Waals forces, ${ }^{7}$ between the tip and the sample surface. ${ }^{4,5}$ Ideally, a repulsive region is not entered. The local gradient of the attractive forces shifts the resonance frequency of the cantilever leading to a change in the phase and amplitude of the oscillation. ${ }^{5,8}$ It is these shifts that are used for sensing the variation in tip-sample distance. The advantage of this mode is the very small disturbance of the sample by the tip; the disadvantage is the limited resolution and the risk of the tip being captured by adsorbed liquid layers ${ }^{6}$ when such layers are present.

At high amplitudes (typically 5-50 nm) the tip will bounce in and out of the force fields, and is generally expected to enter into the repulsive region. ${ }^{6,9}$ Most commonly, it is the brute damping of the amplitude that is used for feedback. This mode, often referred to as the tapping mode, ${ }^{6}$ is particularly advantageous on soft surfaces, since there are almost no lateral forces involved compared to contact-mode operation, i.e., the tip is not dragged over the surface but samples the topography pointwise. In contrast to the true non-contact mode, resolution is not sacrificed since the tip actually goes into contact during each cycle. Atomic resolution has recently been demonstrated in ultrahigh vacuum on the reconstructed $\mathrm{Si}$ (111) $7 \times 7$ surface $^{10}$ using a low swing amplitude of $\sim 1.6 \mathrm{~nm}$. Due to the heavily reduced lateral forces, the tapping tip scheme has naturally had a big impact on the microscopy of soft matter, e.g., biological materials, ${ }^{11}$

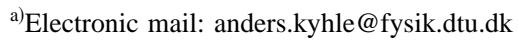

but has also been found well suited to investigate small objects loosely attached to different kinds of planar surfaces. ${ }^{12}$ The applicability of this mode is thus very high, since it is much more versatile compared to the usual contact mode of operation.

The interactions between a steady tip and a surface has been dealt with in a number of publications. ${ }^{4,7,13,14}$ A comprehensive paper has been published by Burnham, Colton, and Pollock ${ }^{15}$ especially devoted to comparing experimental force curves with different possible physical mechanisms. Besides the actual tip-surface interactions, the dynamics of the vibrating cantilever ${ }^{16}$ play a vital role in the behaviour of the tapping tip mode. An oscillating cantilever together with the tip-sample interactions constitutes a highly non-linear system and artifacts related to the dynamics of this system may often be encountered. One commonly observed artifact is a discontinuity on the high frequency side of the cantilever resonance peak, when the tip is in momentary contact [Fig. 2(a)]. By using a simple model for the cantilever-tip-sample system (Fig. 1), where the tip/cantilever is modelled as a mass attached to a massless spring and the surface simply as a spring, Spatz et al. ${ }^{9}$ have in a recent paper shown that this artifact is due to an increase in the resonance frequency due to the elastic interaction with the surface, i.e., an effective increase in the average spring constant of the cantilever. One thus expects that the system may become unstable if the frequency operating point is set on the right-hand side of the cantilever resonance peak.

In this paper we present experimental data which show the diverse properties of the vibrating cantilever/tip/sample system. Our results indicate that attractive forces play a key role in the stability of the tapping tip mode and that they introduce more instabilities than predicted in the model by Spatz et al. ${ }^{9}$ We compare our findings with simulations similar to those published by others, ${ }^{9,17,18}$ taking into account attractive forces, which are especially pronounced when operation takes place in ambient environment. ${ }^{15}$ The attractive forces are included phenomenologically without the intention of simulating the exact functional behaviour of forces with specific physical origin. At the time of preparing this paper we have become aware of similar work by Anc- 


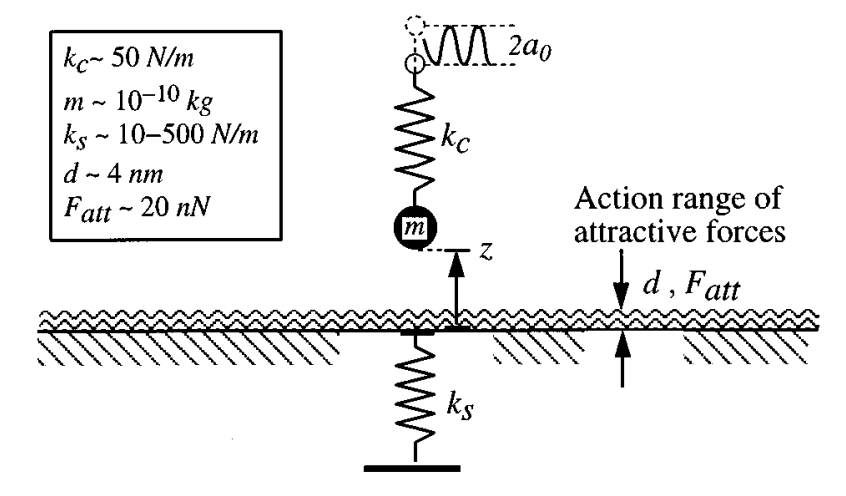

FIG. 1. Model of the tip, cantilever, sample, and their interactions. The cantilever and the tip are modelled as a mass $m$ suspended in a massless spring with the spring constant $k_{c}$. The repulsive surface forces are modelled as a spring $k_{s}$ and the attractive forces as a constant force $F_{\text {att }}$ acting in a specified range $d$. The distance from the tip to where the repulsive forces start acting is $z$, and $a_{0}$ is the amplitude of the driving signal.

zykowski and co-workers ${ }^{18}$ whose conclusions are very similar to ours.

\section{PROCEDURES}

\section{A. Experiments}

The microscope used for the experiments was a Topometrix TMX 2000 Explorer model (a scanning tip system). The cantilevers were of the rectangular bar type with a nominal spring constant of $35-65 \mathrm{~N} / \mathrm{m}$ and nominal dimensions of $220 \times 40 \times(3.2-4.0) \mu \mathrm{m}^{3}$. For consistency, all the measurements presented here were performed using the same cantilever. No tip degradation was observed during the measurements. In order to minimize wear of the tip no or only little scanning took place between the measurements. The resonance frequency $f_{c}$ and the $3 \mathrm{~dB}$ bandwidth $\Delta f_{3 \mathrm{~dB}}$, of the cantilever were measured to $f_{c}=157324 \pm 5 \mathrm{~Hz}$ and $\Delta f_{3 \mathrm{~dB}}=420 \pm 10 \mathrm{~Hz}$, respectively, the quality factor thus being $\mathrm{Q} \approx 375$. For the measurements of the magnitude and phase response (with respect to the driving oscillator) of the cantilever signal an external computer controlled lock-in amplifier was used. The internal oscillator of the lock-in amplifier was used for the modulation signal. The phase difference between the reference signal of the lock-in amplifier and the cantilever response was offset such that continuous phase versus frequency curves could be recorded. The frequency response curves presented here were recorded using the lock-in amplifier for nominal swing amplitudes of 5, 35, and $50 \mathrm{~nm}$. For each amplitude the response was recorded at different average distances between the tip and the sample starting with a recording taken before actual tip-sample contact took place and hereafter at different tip-sample separations with increased damping of the oscillatory motion of the cantilever. The distance between tip and sample was controlled by fixing the dc voltage applied to the $z$-axis piezo. In order to reduce the influence of thermal drift and piezo creep during the measurements we would wait for the system to relax at each set point, and check the distance both before and after each measurement. The nominal swing amplitudes were determined from amplitude versus distance traces, which yield rather linear curves at high amplitudes $(>20$ $\mathrm{nm})$. In this way the necessary amplitude of the modulation signal was determined for the $35 \mathrm{~nm}$ swing. For the other desired amplitudes the modulation signal was scaled linearly.

Spectroscopy traces (amplitude and phase response) with respect to the distance between the sample surface and the fixed end of the cantilever were measured using the microscope's internal software and the signals from the external lock-in amplifier.

Measurements were performed on three different samples: oxidized $n$-doped (111) $\mathrm{Si}\left(\mathrm{SiO}_{x}\right)$, freshly cleaved highly oriented pyrolytic graphite (HOPG), and freshly cleaved muscovite mica.

All experiments were performed in ambient environment in a temperature controlled room. The relative humidity was measured for each experiment with an ordinary hair-sensor hygrometer with an accuracy of $\pm 3 \%$. The samples could not be measured within the same day in the laboratory, but for each sample the measurements were carried out consecutively on the same day, i.e., at the same temperature and humidity.

\section{B. Simulations}

Simulations of the cantilever motion and tip-sample interaction were carried out solving the equation of motion (1) for the model depicted in Fig. 1. The cantilever and tip are modelled as a mass $m$ suspended by a mass less spring with the spring constant $k_{c}$. The repulsive force between the tip and the sample is modelled as a mass less spring with the spring constant $k_{s}$.

The equation of motion for the model system (a driven harmonic oscillator with weak damping) is given by:

$$
\ddot{z}+\frac{\omega_{0}}{Q} \dot{z}+\omega_{0}^{2} z=\frac{1}{m} F(z)+a_{0} \omega_{0}^{2} \sin (\omega t),
$$

where $z$ is the distance between the tip and the point of onset of repulsive forces, $\omega_{0}$ is the angular resonance frequency of the freely swinging cantilever $\left(2 \pi f_{c}\right)$, and $Q$ is the quality factor of the oscillator. $a_{0}$ and $\omega$ are the amplitude and angular frequency of the applied driving signal, respectively. $F(z)$ is the sum of repulsive and attractive forces acting between the tip and the surface. In our simulations $F(z)$ is simply:

$$
F(z)= \begin{cases}-F_{\text {att }}, & 0 \leqslant z<d, \\ -F_{\text {att }}-k_{s} z, & z<0,\end{cases}
$$

where $d$ is the action range of the attractive forces of magnitude $F_{\text {att }}$, and $k_{s}$ is the effective surface "spring constant." Note that $z$ becomes negative when the tip indents into the surface. The equation of motion (1) was integrated using a fourth-order Runge-Kutta routine ${ }^{19}$ with adaptive step size control. Lock-in detection was simulated using the same procedure as used in a real digital lock-in amplifier, i.e., by multiplying the temporal signal with two square waves at the reference frequency but shifted by $90^{\circ}$ in phase. The resulting products are time integrated. The functionalities of the interaction forces are kept simple in order to ease the interpretation. Nevertheless, the $z$ dependence of the re- 


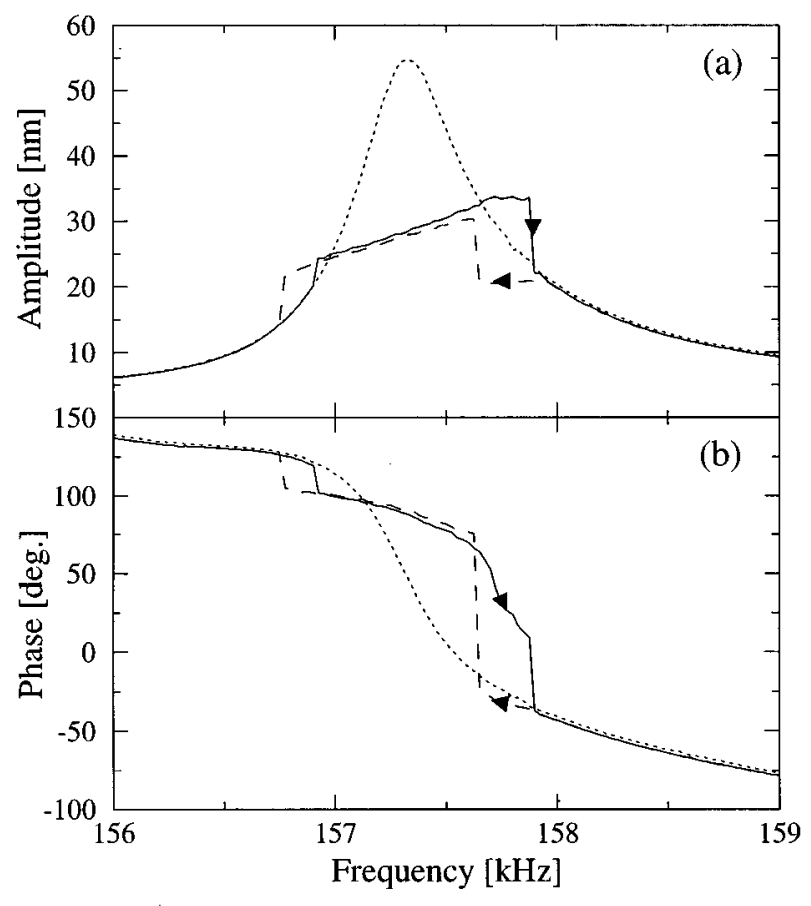

FIG. 2. Measured frequency response of the cantilever: (a) magnitude, (b) phase shift. Dotted line: free swing. Solid line: damped spectrum with positive frequency sweep direction. Dashed line: damped spectrum with negative frequency sweep direction. Characteristic abrupt jumps in the magnitude and phase are observed on both the high- and the low-frequency side of the damped spectrum. The temperature and relative humidity were $T=$ $23.7^{\circ} \mathrm{C}, \mathrm{RH}=34 \%$.

pulsive forces is only a minor simplification of the expected Hertzian ${ }^{17,20}$ distance dependence of the force between a planar surface and a sphere indenting onto it: $F(z) \propto z^{3 / 2}$. This difference does not change the qualitative results of our simulations. The magnitude of the force constant $k_{s}$ was chosen such as to reproduce the experimental data most convincingly. The choice of the abruptness, the action range, and the magnitude of the attractive forces was inspired by the effect of capillary forces, though only simulating these in a rough way, neglecting any hysteresis.

\section{FREQUENCY RESPONSE}

\section{A. Measurements}

Figure 2 shows the frequency response of the cantilever when it is driven such that the nominal free swing amplitude at the top of the resonance peak is about $50 \mathrm{~nm}$. The sample is $\mathrm{SiO}_{x}$. The dotted lines represent the free swing and the solid and dashed lines represent the response when the cantilever has been brought so close to the sample surface that it will go into momentary contact at sufficiently high amplitudes. The solid lines in Fig. 2 represent the damped signal when the frequency sweep goes from low to high frequencies and the dashed lines represent the response when the sweep goes from high to low frequencies. Figure 2(a) shows the magnitude of the cantilever signal and Fig. 2(b) shows the change in phase of the signal when traversing the point of resonance.
Two characteristic features are seen on the curves representing the damped signal, namely an abrupt amplitude increase/decrease at the low frequency (LF) side of the spectrum and at the high frequency $(\mathrm{HF})$ side of the spectrum. Corresponding jumps are observed in the phase response. The jump on the HF side of the spectrum can be attributed to resonance enhancement due to elastic interaction between the tip and the sample, ${ }^{9}$ i.e., the effective spring constant and hence the resonance frequency are increased leading to an enhancement of the swing amplitude for higher frequencies. This mechanism is responsible for the amplitude to exceed the free swing amplitude in an interval to the right-hand side of the free swing curve. From this argument one would expect hysteresis between the HF-side jump in the upward and downward frequency sweeps. One would expect the downward sweep to follow the free swing curve until a point near the intersection with the upward sweep curve. Instead the downward curve is observed to follow a flat path from some point on the free swing curve down to a frequency slightly below the frequency where the free swing curve and the damped upward sweep curve intersect. We will discuss this issue in greater detail below.

The amplitude and phase jump on the LF side of the spectrum which has not previously been reported we believe is caused by the attractive forces between the tip and sample. This will be illustrated in the simulations in the next subsection. Such attractive forces are van der Waals forces, ${ }^{7}$ electrostatic forces (e.g., patch-charge forces ${ }^{15}$ ), and capillary forces induced by adsorbed liquid layers. ${ }^{7}$ Qualitatively, the amplitude jump can be understood in the following way: When the frequency (swept from low frequencies) gets so high that the tip, when at its outermost position, first dips into the action range of the attractive forces, the attractive forces will pull the tip closer to the surface and effectively charge the cantilever with an extra amount of potential energy. This results in the cantilever gaining enough energy to dip even further into the action range of the attractive forces at the end of its next cycle, where it again will gain extra energy, effectively resulting in an abrupt increase in the swing amplitude. The effect of the attractive forces may also be looked upon as an effective decrease of the spring constant of the cantilever and hence of its resonance frequency. The amplitude increase is limited by the repulsive forces acting when the tip and sample come into close contact. The jump may occur at a lower frequency due to fluctuations, i.e., the cantilever is a high $Q$ resonance circuit that is easily disturbed. The hysteresis of the LF-side jump with respect to the upward and downward sweeps is readily understood since for the up-going sweep the tip needs to come close to the action range of the attractive forces, whereas for the down-going sweep it is a question of how far the energy input from the driving oscillator can sustain the large amplitude.

\section{B. Simulations}

In Fig. 3 is shown the results from a set of simulations. The dotted curves represent the free swing of the cantilever. The curves labelled $\mathrm{R}$ and $\mathrm{A}$ are when pure repulsive or pure attractive forces are present, respectively. The thick curves 


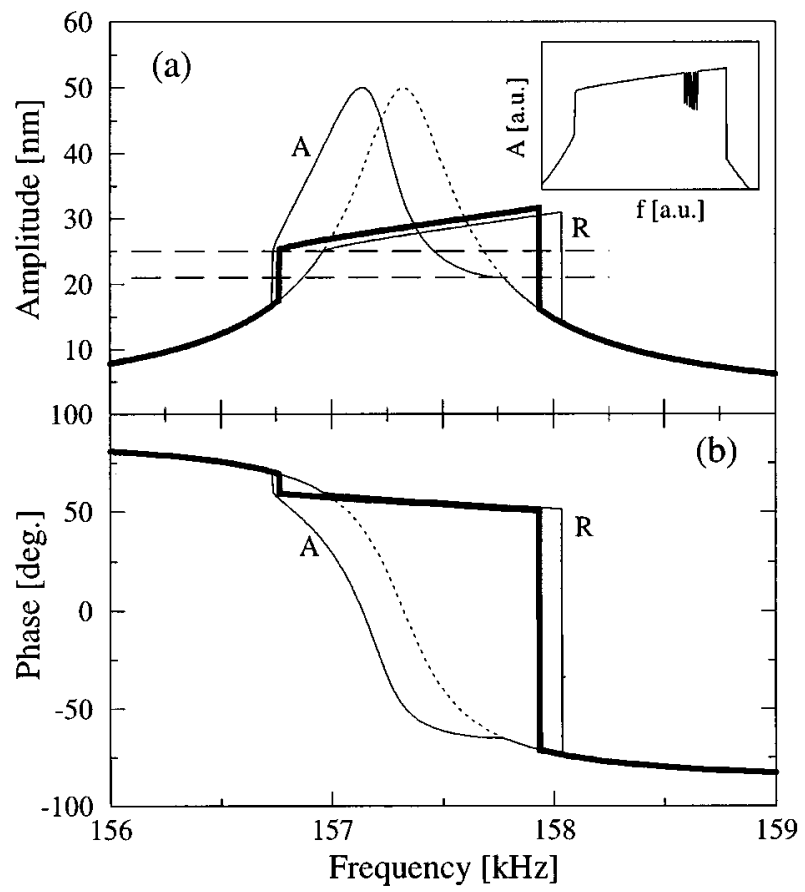

FIG. 3. Simulated amplitude (a) and phase (b) response curves. Repulsive forces only $\left(k_{s}=25 \mathrm{~N} / \mathrm{m}\right)$ for the curves labelled $\mathrm{R}$, and attractive forces only for the curves labelled A $\left(F_{\text {att }}=20 \mathrm{nN}, d=4 \mathrm{~nm}\right)$. The thick solid curves are the results when both attractive and repulsive forces are present. The dotted curves are for the free swing. The relative phase is offset by $90^{\circ}$. The inset shows spikes in the simulation due to switching between the metastable solutions of the A curve and the thick curve. For clarity, occasional spikes have been removed from the presented data.

are the results when both attractive and repulsive forces act according to (2). The constants used were $k_{s}=25 \mathrm{~N} / \mathrm{m}$, $F_{\text {att }}=20 \mathrm{nN}$, and $d=4 \mathrm{~nm}$. The lower and upper horizontal dashed lines represent the points of onset of attractive and repulsive forces, respectively. The phase difference has been offset $90^{\circ}$ in order to obtain continuous curves. It is observed that the calculated curves qualitatively resemble the experimental data. One small difference is the magnitude of the jump on the LF side of the peak: the shift in phase is notably larger in the experimental case $\left(17.6^{\circ}\right.$, upward sweep, $21.6^{\circ}$, downward) in comparison with the simulation $\left(10.1^{\circ}\right)$. In addition the amplitude shift is somewhat smaller in the experiment $(4.3 \mathrm{~nm}$, upward, $7.6 \mathrm{~nm}$, downward) when compared to the simulation $(8 \mathrm{~nm})$. This may partly be explained by the following argument. From Fig. 3(a) it can be seen that the closer to $f_{c}$ the jump occurs, the smaller the jump in amplitude will be (due to the repulsive forces). Comparing the dashed curve and the A curve in Fig. 3(b) it is seen that the opposite is the case for the jump in phase. In the simulation the system is perturbed appreciably every time the frequency is stepped during the sweep. This results in the cantilever amplitude to beat with a frequency equal to the difference between the resonance frequency and the driving frequency. An amplitude interval around the steady state value is thus swept in this way for each step. This sometimes results in switching between two metastable solutions such as can be seen on the inset of Fig. 3(a). For clarity the curves in Fig. 3 have been stripped for a few of these events. Be- cause of this beating behaviour the point where the jump on the LF side occurs will be very close to where the upper solution becomes more stable than the lower solution, which is at a lower frequency than where the free swing curve intersects the horizontal dashed line representing the onset of attractive forces. Comparing with the experiment this point will be somewhere near the jump in the downward sweep (Fig. 2). However, there will also be physical reasons for the observed differences involving the magnitude and functionality of the attractive forces.

It is important to note that when the tip first starts interacting with the attractive forces [(LF side of the thick curve of Fig. 3(a)] it will "tap" right through the action range of the attractive forces and enter the region of repulsive forces. Also noteworthy is our observation that the magnitude of the amplitude/phase jump on the LF side of the A curve in Fig. 3(a) only depends slightly on the action range of the attractive forces but strongly on the magnitude. From the A curve in Fig. 3(a) we can learn why the experimental curve for the downward sweep in Fig. 2(a) on the HF side follows a flat path until the tip abruptly starts interacting elastically with the sample. The reason is that in this region the response follows the corresponding A curve for the experiment, i.e., the tip interacts with the sample only through attractive forces. The chosen magnitude for the attractive forces of 20 $\mathrm{nN}$ is within the correct range for measured and theoretically predicted forces. ${ }^{7,14,15,21,22}$ The surface spring constant of 25 $\mathrm{N} / \mathrm{m}$ is also consistent with values calculated and used by others. ${ }^{9,21,23}$ If one were to reproduce the damped curves in Figs. 2(a) and 3(a) using Hertz's theory ${ }^{20}$ for the repulsive forces, a Young's modulus of about $E \approx 3 \mathrm{GPa}$ would be required, assuming identical tip/sample materials with a Poisson's ratio of about 0.35 , and a tip radius of about 20 $\mathrm{nm}$. This value is on the low side for $\mathrm{Si}$ and $\mathrm{SiO}_{x}: E_{\mathrm{Si}} \approx 180 \mathrm{GPa}, E_{\mathrm{SiO}} \approx 60 \mathrm{GPa}^{4}$

\section{Sample and amplitude dependence}

Figure 4 shows three sets of amplitude versus frequency response curves (upward sweeps) on the $\mathrm{SiO}_{x}$ sample for nominal free swing amplitudes of $50 \mathrm{~nm}$ (top), $35 \mathrm{~nm}$ (middle), and $5 \mathrm{~nm}$ (bottom). It is readily observed that the shape of the damped curves depends strongly on the chosen free swing amplitude and on the amount of damping. For the $50 \mathrm{~nm}$ amplitude curves (A-E) we observe that elastic tapping does not occur for low degrees of damping: The curve $\mathrm{B}$ has a negative slope. The curve $\mathrm{C}$ shows the signature of elastic tapping (positive slope) for frequencies up to a point slightly above $f_{c}$ where there is an abrupt transition. The curves for higher degrees of damping all show the signature of elastic tapping. At $35 \mathrm{~nm}$ free swing amplitude $(\mathrm{F}-\mathrm{H})$ the elastically resonance enhanced tapping does only appear for dampings by more than $40 \%$, and from curve $\mathrm{H}$ it is seen that even for this high degree of damping a transition to the regime of "attractive" damping occurs near $f_{c}$. For amplitudes around $5 \mathrm{~nm}(\mathrm{I}-\mathrm{L})$ we neither observe a jump on the LF side of the spectrum (attractive amplitude enhancement) nor a jump on the HF side of the spectrum (repulsive elastic amplitude enhancement). Furthermore, we do not observe a flat top on the damped curves. 


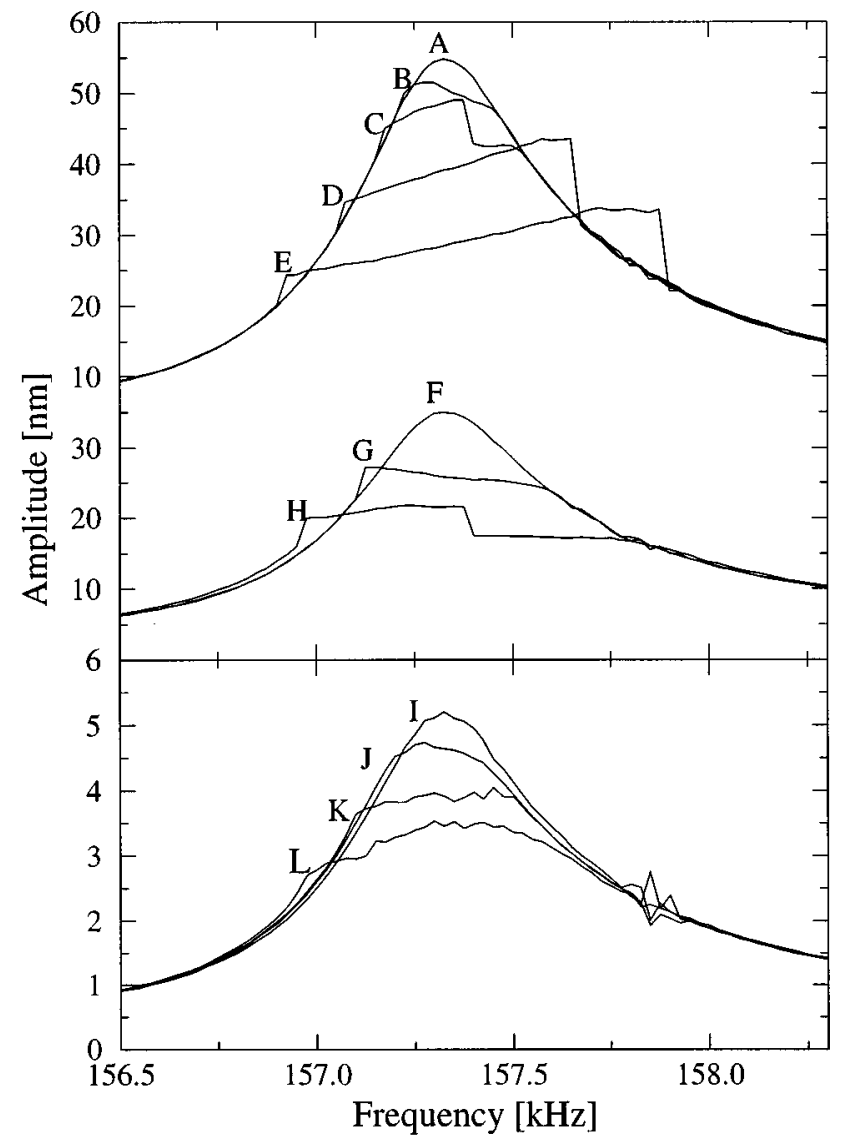

FIG. 4. Frequency response curves for the $\mathrm{SiO}_{x}$ sample for different degrees of damping at three different nominal free swing amplitudes: $50 \mathrm{~nm}$, top, 35 $\mathrm{nm}$, middle, $5 \mathrm{~nm}$, bottom.

For the mica sample and the HOPG sample the behaviour is somewhat similar to that shown for the $\mathrm{SiO}_{x}$ sample. For HOPG only at a free swing amplitude of $50 \mathrm{~nm}$ and damping of more than $50 \%$ we can observe elastic tapping, and only for damping by more that $70 \%$ is there no switch back to the regime of damping due to attractive forces (negative slope regime). Otherwise, we do not observe the signature of elastic tapping on either mica or HOPG samples at the measured amplitudes. It would be interesting for each type of sample to examine at which levels of free swing amplitude and damping elastic tapping sets in. Work in this direction is in progress. Amplitude versus frequency response curves for HOPG and mica are shown in Figs. 5(a) and 5(b), respectively, with the corresponding phase shifts plotted in insets. The jump due to attractive forces is observed on all the damped curves. The negative slope of the damped curves is observed to be a little bit steeper for HOPG when compared with mica. The temperature and the relative humidity during the measurements were $T=23.2{ }^{\circ} \mathrm{C}, \mathrm{RH}$ $=38 \%$ for HOPG and $T=22.8{ }^{\circ} \mathrm{C}, \mathrm{RH}=37 \%$ for mica.

\section{SPECTROSCOPY}

In Fig. 6 is shown representative amplitude versus distance curves for the tip sample approach on the $\mathrm{SiO}_{x}$ sample. The free amplitude at the resonance frequency $f_{c}$ was 35

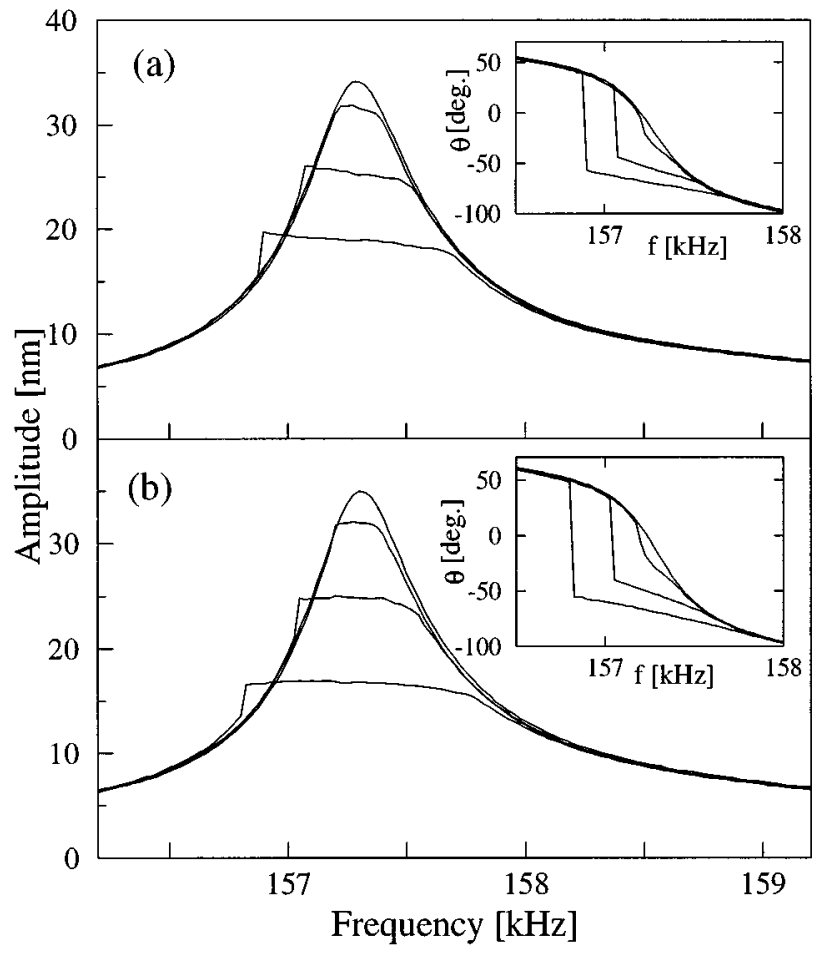

FIG. 5. Frequency response curves for the HOPG sample (a) and the mica sample (b) for different degrees of damping. The cantilever free swing amplitude is $35 \mathrm{~nm}$. The insets show the corresponding phase shifts.

$\mathrm{nm}$. The distance is between the equilibrium position of the tip and an arbitrary reference position near the sample surface. The solid curve shows the response when the cantilever is excited at a frequency $200 \mathrm{~Hz}$ below $f_{c}$. It is seen that the amplitude increases abruptly at the point where the tip starts interacting with the sample and hereafter decreases almost linearly with the distance: The amplitude is first enhanced due to attractive interaction and thereafter damped due to the repulsive interaction. The thick dotted curve is when the driving frequency is the resonance frequency. In this case

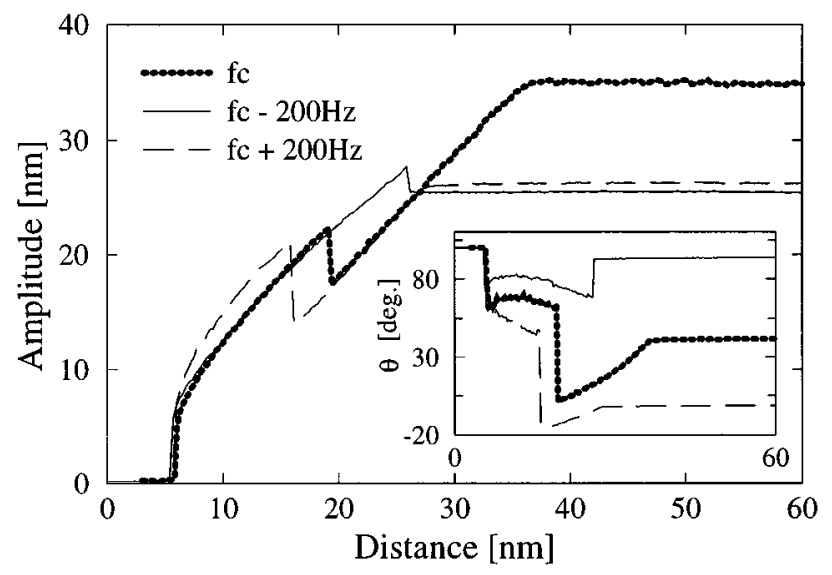

FIG. 6. Amplitude vs distance traces performed on the $\mathrm{SiO}_{x}$ sample with a free swing amplitude at $f_{c}$ of about $35 \mathrm{~nm}$. Data taken at $f=f_{c}$, thick dotted line, and at $f_{c}-200 \mathrm{~Hz}$, solid line, and $f_{c}+200 \mathrm{~Hz}$, dashed line. The inset shows the corresponding phase variations. 


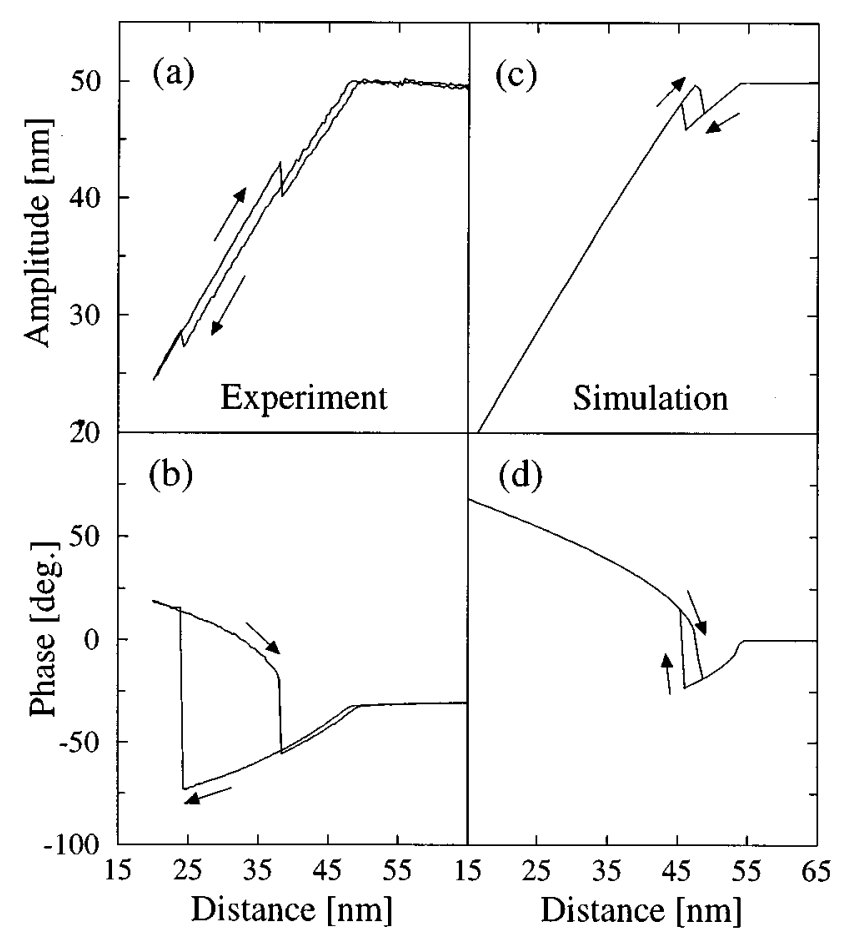

FIG. 7. General forward/reverse amplitude and phase vs distance trace when $f=f_{c}$ (HOPG sample, $50 \mathrm{~nm}$ amplitude). (a) and (b) Experimental data; (c) and (d) simulation.

there is no amplitude enhancement until at a certain distance where there is a jump. This point is where the point of operation goes from being on the slightly damped curves with negative slope in Fig. 4 to the more heavily damped curves with positive slope, i.e., there is a jump from attractive damping to repulsive damping. That the jump is associated with a transition from attractive dominated damping to repulsive dominated damping is substantiated by the corresponding phase versus distance curve plotted in the inset of Fig. 6. In addition to the jump in the phase, the slope of the $\theta(d)$ curve changes at the transition point. In the attractive mode the phase decreases as the damping is increased, and in the repulsive mode the phase difference increases as the distance $d$ is reduced. On the dashed curve the frequency was set 200 $\mathrm{Hz}$ above resonance. This curve has the same qualitative feature as when $f=f_{c}$ except that the jump in amplitude is considerably larger. Comparing with the frequency sweeps in Fig. 2(a) one can see that this jump occurs in the region on the HF side of the spectrum in Fig. 2 where the upward and downward sweeps do not coincide. In all three cases close to the sample the oscillation is killed abruptly. We believe that this is due to the tip being captured by an adsorbed liquid layer (known to be present on some surfaces ${ }^{14,24}$ ) when the amplitude becomes smaller than a critical value. ${ }^{6}$

In general, the position of the point of switching between attractive and repulsive tapping shows hysteresis with respect to the scan direction (approach or retraction). This includes both at, below and above the resonance frequency. In Fig. 7(a) and 7(b) are shown approach and retraction curves of the amplitude and phase. The curves are general representatives from traces taken at $f=f_{c}$ for a configuration of
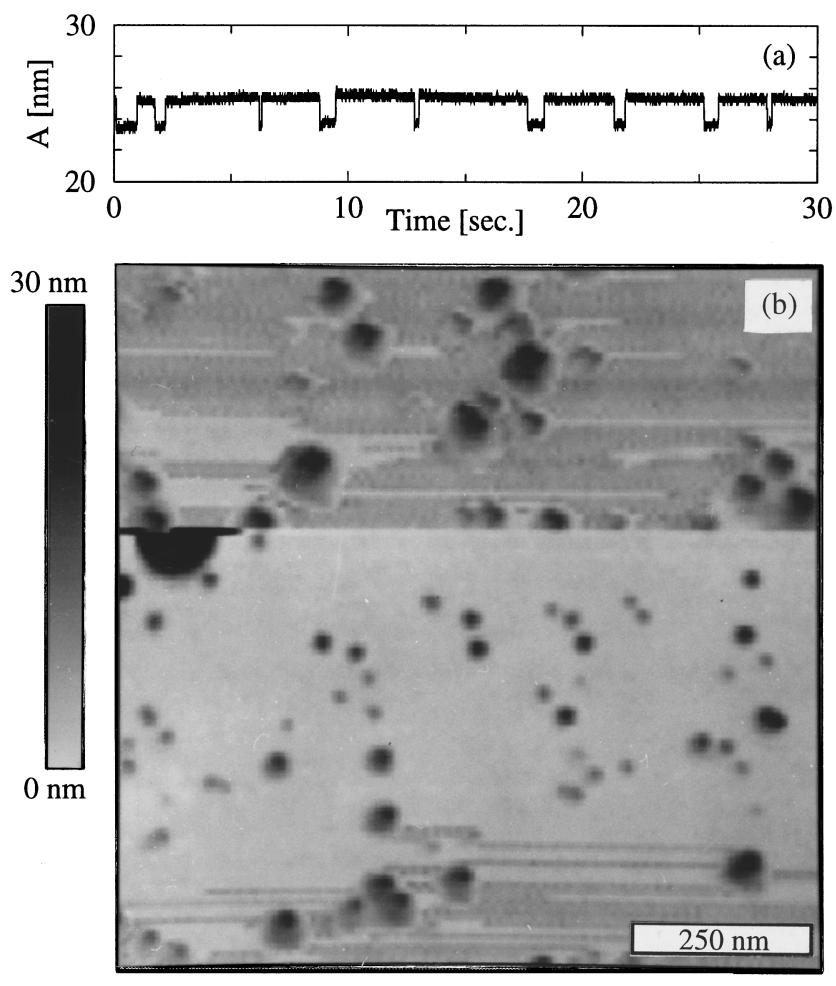

FIG. 8. (a) Stochastic amplitude switching when the cantilever-sample distance is close to the instability point $\left(\mathrm{SiO}_{x}\right.$ sample, $\left.a_{\text {free }}=35 \mathrm{~nm}\right)$. (b) Example of the influence on imaging: shifts in apparent resolution and sample height level ( $\mathrm{Cu}$ clusters on a $\mathrm{SiO}_{x}$ surface).

sample and free swing amplitude that displays both the attractive and the repulsive elastic damping (HOPG at $50 \mathrm{~nm}$ swing). The width of the observed hysteresis is seldomly reproduced exactly and varies between different positions on the sample. In Fig. 7(c) and 7(d) is shown the corresponding amplitude and phase versus distance curves obtained by numerical simulation. The qualitative features, i.e., the presence of hysteresis and the change of slope of the phase versus distance curve, are reproduced, although the width of the hysteresis loop and the absolute shift in phase difference do not fit the experimental data precisely.

\section{DISCUSSION}

The observed switching behaviour can influence image acquisition, and this is the reason why we initiated these studies. As shown in Fig. 8(a), when the system is left in a state close to the switching point and the feedback is disabled, random switching in amplitude and phase can be observed (random telegraph noise). When switching occurs during image acquisition this results in sudden shifts in the apparent resolution and in the height of the sample. An example is shown in Fig. 8(b), which is an image of $\mathrm{Cu}$ clusters on a $\mathrm{SiO}_{x}$ surface. $^{25}$ In comparison to the central part of the image, the resolution is poor (the tip seems big) in the top and bottom parts of the image and the height level of the underlying surface is shifted upwards. These effects are often interpreted as if the tip had picked up material, e.g., a particle. In the shown example we are confident that this is not the case, since switching along and between individual scan 
lines is seen. If (in amplitude feedback) the temporal cantilever signal is monitored on an oscilloscope, the switching between the two curves will be observed as jumps in the phase of the signal. Operation on either of the attractive or repulsive dominated branch of the spectroscopy curves can be promoted by changing the driving frequency to a little bit above or below resonance, respectively, as can be seen from Fig. 6.

For the feedback loop in any system to work properly it must be fed with a signal that varies monotonously with the regulated parameter, which in the AFM is the tip sample separation. A single or a few jumps in the signal versus distance curve (e.g., Fig. 6) will not necessarily, as shown in Fig. 8, spoil the operation of the feedback loop; but, in the case that the signal versus distance curve changes slope, the feedback loop will be unable to operate correctly. In, e.g., a thermal regulation this can result in thermal runaway. In the AFM the result (worst case) will be a tip crash. Such a change in slope is accompanying the jump in the phase versus distance curves shown in Fig. 6 (inset) and in Fig. 7(b). Feedback based on phase detection thus cannot be recommended on $\mathrm{SiO}_{x}$ samples or in general on samples where attractive and elastically repulsive tip-sample interactions compete. Furthermore in the repulsive regime the phase changes only very little with distance (see inset in Fig. 6) and is thus not well suited for use in feedback. However, on samples, like mica and possibly soft samples in general, where elastic tapping is not observed, phase detection may be used safely. In general the magnitude of attractive forces (e.g., van der Waals or capillary forces) must be expected to decrease with decreasing tip size ${ }^{21}$ i.e., the sharper the tip, the smaller the magnitude and probability of switching.

We have observed that the apparent imaging resolution of isolated $\mathrm{Cu}$ particles on $\mathrm{SiO}_{x}$ surface in the attractive mode (in ambient atmosphere) is worse than in the repulsive mode. This observation, however, does not enable us to draw a general conclusion about the resolution of the attractive mode. Though the attractive (non-elastic) tapping is dominant on both HOPG and mica, the origin (and range) of the interactions may be different from those on $\mathrm{SiO}_{x}$ (and $\mathrm{Cu}$ ). Additionally the apparent resolution must be expected to depend on the sample topography, i.e., a ripple with a nanometer sized wavelength may be resolved but an isolated particle of the same size may appear enlarged.

Both our experimental and numerical spectroscopy results agree well with those published recently by Anczykowski and co-workers ${ }^{18}$ who in the simulations used a potential calculated from a molecular dynamics approach, and for their experiments used (100) Si substrates and a cantilever with a resonance frequency of about $310 \mathrm{kHz}$ and swing amplitudes of about $40 \mathrm{~nm}$.

Though we have shown that attractive forces constitute the dominant type of interaction in tapping tip force microscopy of some samples, we have not discussed the detailed mechanisms involved. Additionally, we have not dealt with any visco-elastic forces from tip-sample deformation, viscous damping by adsorbed contamination layers (hydrocarbons and water), and hysteresis in the capillary forces between the tip and such layers. Also wetting by the tip and the sample by tip/sample atoms during contact has not been considered. For example, our model cannot reproduce very well the damped frequency spectra recorded on the mica sample where elastic tapping is not observed. Another damping mechanism probably involving energy dissipation is needed to account for the observed non-elastic damping.

\section{CONCLUSIONS}

In summary we have observed the impact of attractive forces on the dynamics of a vibrating cantilever in an atomic force microscope. In addition to the instability in the frequency spectrum which can be assigned to repulsive forces of elastic type, the attractive forces also give rise to an instability. Furthermore, we find for the samples measured here that the most dominant forces involved in tapping tip microscopy are the attractive forces. A jump and a sign reversal in the slope of the phase versus distance curves indicate a transition between the attractive forces being the dominant damping mechanism and the repulsive forces being the dominant damping mechanism. This jump can also be observed in the frequency response of the damped system. Under certain, but in experimental situations very common, combinations of the nature of the tip and sample, cantilever swing amplitude, and the damping set point, this transition occurs close to the operating point and gives rise to switching in the amplitude and phase of the oscillating cantilever. This can be observed during image acquisition as jumps in the apparent resolution and in the height level of the sample. These switching effects naturally occur only on samples where repulsive elastic forces are present, i.e., on hard surfaces.

\section{ACKNOWLEDGMENTS}

This work was supported by the Danish Ministries of Industry and Research within the framework of CNASTCentre for Nanostructures.

${ }^{1}$ G. Binnig, C. F. Quate, and C. Gerber, Phys. Rev. Lett. 56, 930 (1986).

${ }^{2}$ P. K. Hansma, V. B. Elings, O. Marti, and C. E. Bracker, Science 242, 209 (1988).

${ }^{3}$ E. Meyer Prog. Surf. Sci. 41, 3 (1992).

${ }^{4}$ D. Sarid, Scanning Force Microscopy With Applications to Electric, Magnetic and Atomic Forces (Oxford University Press, New York, 1994).

${ }^{5}$ Y. Martin, C. C. Williams, and H. K. Wickramasinghe, J. Appl. Phys. 61, 4723 (1987).

${ }^{6}$ Q. Zhong, D. Inniss, K. Kjoller, and V. B. Elings, Surf. Sci. Lett. 209, L668 (1993).

${ }^{7}$ J. Israelachvili, Intermolecular and Surface Forces, 2nd ed. (Academic, 1991).

${ }^{8}$ D. Anselmetti, R. Lüthi, E. Meyer, T. Richmond, M. Dreier, J. E. Frommer, and H.-J. Güntherodt, Nanotechnology 5, 87 (1994).

${ }^{9}$ J. P. Spatz, S. Sheiko, M. Möller, R. G. Winkler, P. Reineker, and O. Marti, Nanotechnology 6, 40 (1995).

${ }^{10}$ R. Erlandsson, L. Olsson, and P. Mårtensson, Phys. Rev. B 54, 8309 (1996).

${ }^{11}$ K. Umemura, H. Arakawa, and A. Ikai, Jpn. J. Appl. Phys. 32, L1711 (1993).

${ }^{12}$ T. Junno, K. Deppert, L. Montelius, and L. Samuelson, Appl. Phys. Lett. 66, 3627 (1995).

${ }^{13}$ A. L. Weisenhorn, P. K. Hansma, T. R. Albrecht, and C. F. Quate, Appl. Phys. Lett. 54, 2651 (1989).

${ }^{14}$ M. Binggeli and C. M. Mate, Appl. Phys. Lett. 65, 415 (1994).

${ }^{15}$ N. A. Burnham, R. J. Colton, and H. M. Pollock, Nanotechnology 4, 64 (1993). 
${ }^{16}$ G. Y. Chen, R. J. Warmack, T. Thundat, D. P. Allison, and A. Huang, Phys. Rev. Sci. Instrum. 65, 2532 (1994).

${ }^{17}$ S. Sarid, J. Chen, and R. K. Workman, Comput. Mater. Sci. 3, 475 (1995).

${ }^{18}$ B. Anczykowski, D. Krüger, and H. Fuchs, Phys. Rev. B 53, 15485 (1996).

${ }^{19}$ W. H. Press, S. A. Teukolsky, W. T. Vetterling, and B. P. Flannery, Numerical Recipes, 2nd ed. (Cambridge University Press, Cambridge, 1992.

${ }^{20}$ K. L. Johnson, K. Kendall, and A. D. Roberts, Proc. R. Soc. London Ser. A 324, 301 (1971)

${ }^{21}$ N. A. Burnham and R. J. Colton, J. Vac. Sci. Technol. A 7, 2906 (1989).
${ }^{22}$ F. O. Goodman and N. Garcia, Phys. Rev. B 43, 4728 (1991).

${ }^{23}$ M. Radmacher, R. W. Tillmann, and H. E. Gaub, Biophys. J. 64, 735 (1993).

${ }^{24}$ K. Hermansson, U. Lindberg, B. Hök, and G. Palmskog, in TRANSDUCERS '91. 1991 International Conference on Solid-State Sensors and Actuators. Digest of Technical Papers, Cat. No. 91CH2817-5 (IEEE, New York, 1991), pp. 193-196.

${ }^{25}$ A. H. Sørensen, A. Kühle, L. Theil Hansen, H. Busch, L. Jin Christiansen, J. Mikkelsen, N. Herholdt-Rasmussen, K. A. Mørch, and J. Bohr, Z. Phys. D (to be published). 